\title{
Formulación y propiedades de biopolímero de recubrimiento para semillas de maní (Arachis hypogaea L.)
}

Montoya, P., Cosiansi, J., Grasso, F. y Melchiorre, M.

DOI:10.31047/1668.298x.v38.n1.30254

\section{RESUMEN}

En este trabajo se presenta el diseño de una biopelícula de recubrimiento biodegradable, formulada con macromoléculas naturales como almidón, proteína y glicerol para ser aplicada sobre semillas de maní, a fin de aumentar la resistencia del tegumento y favorecer la fluidez en la siembra. Se ensayaron diversas formulaciones variando la concentración y tipo de los almidones. Dos formulaciones, una a base de almidón de maíz y otra de almidón de mandioca, fueron caracterizadas en su reología y en las biopelículas se determinaron propiedades de barrera, hidratación y solubilidad en agua. Aplicadas sobre semillas se evaluaron sus características tecnológicas como espesor de capa, capacidad de absorción-desorción de humedad y su aporte a la fluidez y resistencia al daño mecánico. Las semillas recubiertas con el biopolímero constituido con $3 \%$ de almidón de mandioca evidenciaron mayor resistencia mecánica y fluidez y buenas propiedades de barrera. Este trabajo aporta una formulación para el recubrimiento biopolimérico de semillas de maní, que al estar constituido por sustancias naturales de grado alimenticio y biodegradables, posee potencialidad de implementación en el marco de la agricultura sustentable.

Palabras clave: semillas; recubrimiento; almidón; mandioca.

Montoya, P., Cosiansi, J., Grasso, F. and Melchiorre M., 2021. Formulation and properties of coating biopolymer for peanut seeds (Arachis hypogaea L.). Agriscientia 38: 15-27

\section{SUMMARY}

This work shows a biodegradable coating biofilm design formulated with natural macromolecules such as starch, proteins, and glycerol, for its application on peanut seeds, to increase tegument resistance and fluency in sowing. Several formulations were assayed varying the concentration and types of starch. Two formulations, one based on corn starch and the other on cassava starch, were characterized in their rheology and their barrier, hydration and water 
solubility properties were determined. In coated seeds, biofilm technological characteristics, such as layer thickness, moisture absorption-desorption, contribution to fluency and mechanical damage resistance were tested. Seeds coated with biopolymer made up of $3 \%$ cassava starch showed increased mechanical resistance and enhanced fluency and barrier properties. This work provides a formulation for the biopolimeric coating of peanut seeds, which being composed of natural, food grade and biodegradable substances, has the potential to be implemented in sustainable agriculture.

Keywords: seeds; coating; starch; cassava.

Montoya, P. (ORCID: 0000-0002-0722-2808) y Grasso, F. (ORCID: 0000-0002-2186-8437): Universidad Nacional de Córdoba, Facultad de Ciencias Exactas, Físicas y Naturales. Av. Vélez Sarsfield 1611 Ciudad Universitaria, Córdoba, Argentina. Cosiansi, J. (ORCID: 0000-00020324-4428): Universidad Nacional de Córdoba, Facultad de Ciencias Agropecuarias. Ing. Agr. Felix Aldo Marrone 746, Ciudad Universitaria, Córdoba, Argentina. Melchiorre, M. (ORCID: 0000-0003-3113-0679): Universidad Nacional de Córdoba, Facultad de Ciencias Exactas, Físicas y Naturales. Av. Vélez Sarsfield 1611 Ciudad Universitaria, Córdoba, Argentina. Instituto Nacional de Tecnología Agropecuaria (INTA), Instituto de Fisiología y Recursos Genéticos Vegetales, Unidad de Estudios Agropecuarios. Av. 11 de Septiembre 4755, Córdoba, Argentina. Correspondencia a: melchiorre.mariana@inta.gob.ar

\section{INTRODUCCION}

Argentina se ha consolidado como el mayor exportador mundial de maní y la excelencia de sus productos es lo que le ha dado prestigio internacional. La zona manisera, conformada por el sur de las provincias de Córdoba y San Luis, norte de La Pampa y el oeste de Buenos Aires presenta condiciones agroecológicas excelentes para el desarrollo del cultivo. Con una producción en 2019 de 1316500 t y rendimientos promedio de 44,2 qq.ha-1 en caja, Argentina concentra casi el $90 \%$ de la producción primaria y la totalidad del proceso industrial de sus derivados, tales como aceite, pellets y harina; y considerando solo el volumen de exportación, la provincia de Córdoba se ubica en tercer lugar, detrás de India y Estados Unidos (Bolsa de Cereales de Córdoba, 2019; Cadena de maní, 2019).

Una práctica común previa a la siembra, es la aplicación de tratamientos fitoterápicos a las semillas. El procedimiento tradicional consiste en recubrirlas con una mezcla de productos químicos disueltos en agua. Debido a las tensiones generadas por la humectación y secado en la superficie, usualmente se producen deterioros en la integridad del tegumento de las semillas (pelado) lo que genera falta de uniformidad en la distribución del tratamiento y ulterior impacto en la emergencia, sobrevivencia de las plántulas y rendimiento (Bongiovanni, Troilo y Pedelini, 2012).

Una alternativa para evitar el pelado de las semillas y garantizar la distribución uniforme de los fitoterápicos es la aplicación de polímeros de recubrimiento.

Esta tecnología permite combinar diversos compuestos como fungicidas, insecticidas, micro y macro nutrientes y suplementar con promotores de crecimiento y fitohormonas; y en algunos casos, incorporar productos en polvo. La aplicación de estos polímeros de recubrimiento tiene como principal función, vehiculizar y uniformizar la adición de sustancias y disminuir las pérdidas de semillas por roturas y pelado durante las operaciones de la siembra. Además, permite incrementar la fluidez de las semillas en su paso por la sembradora; de esta manera contribuye a la precisión en la descarga en la línea de siembra (Bongiovanni et al., 2012).

En la elaboración de recubrimientos para semillas, es factible utilizar tanto moléculas 
orgánicas naturales, como naturales químicamente modificadas o de síntesis. En el mercado de las semillas, las compañías mantienen estas fórmulas como secretos industriales y las revisan de modo permanente a fin de mejorar sus atributos tecnológicos. Entre las biomoléculas, las propiedades plastificantes del almidón han sido valoradas desde hace muchos años, principalmente en la industria textil, aunque su incorporación para constituir biopelículas es más reciente. El uso del almidón en diversos tipos de películas para envases y recubrimientos, es una alternativa de sustitución a los componentes de síntesis o derivados del petróleo y su empleo ha estado fuertemente motorizado por la diversidad de fuentes de las que puede obtenerse, entre los que se cuentan tanto cereales (maíz y arroz), como tubérculos y raíces (papa y mandioca) (Willet, 2009). Los almidones de orígenes diversos poseen diferentes contenidos porcentuales de amilosa y de amilopectina y, en consecuencia, diferentes niveles de ramificación y entramado en su estructura. Por ello, es posible encontrar diversas propiedades viscoeláticas en las mezclas de las que forman parte (Pérez y Bertoft, 2010). Una importante ventaja de su empleo en la formulación de biopelículas de recubrimiento es que constituyen recursos naturales renovables e inherentemente biodegradables por un conjunto amplio, ubicuo y diverso de microrganismos capaces de utilizarlos como fuente de carbono.

En el presente trabajo se describen las etapas de diseño, evaluación y selección de un formulado para recubrimiento de semillas de maní a base de una mezcla de componentes de grado alimenticio, biodegradables y de bajo costo. El objetivo de este trabajo fue conseguir un recubrimiento biopolimérico que aumente la fluidez y resistencia mecánica de las semillas a la manipulación durante la clasificación y la siembra, asegurando las condiciones óptimas de intercambio de nutrientes, humedad y oxígeno necesarios para la aptitud agronómica de las semillas de maní.

\section{MATERIALES Y MÉTODOS}

\section{Materiales}

Para el formulado de los biopolímeros se ensayaron almidones de maíz (Zea mays L.) y mandioca (Manihot esculenta Crantz), colágeno parcialmente hidrolizado comercial y glicerol, todos los componentes de grado alimenticio. Se incluyó azul ácido, un colorante hidrosoluble de grado técnico a fin de verificar la uniformidad en la distribución de los biopolímeros sobre las semillas. Las formulaciones se aplicaron sobre semillas de maní (Arachys hypogaea L.) Cv Granoleico calibre 6-7.

Para la operación de recubrimiento se utilizó una paila rotativa a escala laboratorio con un recipiente de $1,5 \mathrm{~L}$ de volumen máximo de carga, que posee tres deflectores de $0,5 \mathrm{~cm}$ de altura, para asegurar el movimiento envolvente del contenido con una velocidad máxima de giro de 50 rpm.

\section{Composición de los recubrimientos biopoliméricos}

Los componentes y proporciones empleadas para los formulados se muestran en la Tabla 1. Además de las seis formulaciones propuestas en este trabajo, se usó a los fines comparativos, el polímero comercial Becker Underwood® cuya constitución para el uso se logra mediante la mezcla de sus dos componentes en partes iguales.

\section{Constitución de los biopolímeros}

Para $100 \mathrm{~mL}$ de mezcla, a $85 \mathrm{~mL}$ de agua destilada, se le agregó el glicerol y se agitó durante 30 min a $50 \pm 1{ }^{\circ} \mathrm{C}$; posteriormente se agregó el colágeno previamente hidratado en $5 \mathrm{~mL}$ de agua y se agitó durante otros 30 min a igual temperatura. Finalmente, se incorporó el almidón previamente dispersado en $10 \mathrm{~mL}$ de agua y se elevó la temperatura a $90 \pm 2{ }^{\circ} \mathrm{C}$ o $70 \pm 2{ }^{\circ} \mathrm{C}$, según las

Tabla 1. Composición de los biopolímeros a base de almidón de maíz y mandioca ensayados para recubrimiento (R)

\begin{tabular}{lccccc}
\hline Recubrimiento (R) & $\begin{array}{c}\text { Almidón de maíz } \\
(\% \mathrm{p} / \mathrm{v})\end{array}$ & $\begin{array}{c}\text { Almidón de } \\
\text { mandioca }(\% \mathrm{p} / \mathrm{v})\end{array}$ & Colágeno $(\% \mathrm{p} / \mathrm{v})$ & $\begin{array}{c}\text { Glicerol } \\
(\% \mathrm{p} / \mathrm{v})\end{array}$ & Colorante $(\% \mathrm{p} / \mathrm{v})$ \\
\hline R_1 & 10 & - & 0,09 & 2,5 & 0,2 \\
R_2 & 5 & - & 0,09 & 2,5 & 0,2 \\
R_3 & 3 & - & 0,09 & 2,5 & 0,2 \\
R_4 & 5 & - & 0,045 & 1,25 & 0,2 \\
R_5 & - & 3 & 0,09 & 2,5 & 0,2 \\
R_6 & - & 5 & 0,09 & 2,5 & 0,2 \\
\hline
\end{tabular}


mezclas se formularon con almidón de maíz o mandioca respectivamente. Por último, se adicionó $0,2 \% \mathrm{p} / \mathrm{v}$ de azul ácido y se enfrió a $50 \pm 2{ }^{\circ} \mathrm{C}$ o $25 \pm 2{ }^{\circ} \mathrm{C}$

\section{Caracterización de los biopolímeros}

\section{Viscosidad}

Para determinar la viscosidad se utilizó un reómetro AntonPaar, modelo Physica MCR 301 (Alemania, 2006). De acuerdo con los valores de viscosidad obtenidos de modo exploratorio con un viscosímetro capilar de Ostwald, se decidió aplicar a los fluidos fuerzas rotacionales con geometría de platos paralelos de $50 \mathrm{~mm}$ de diámetro y distancia entre ellos de $1 \mathrm{~mm}$. La velocidad de rotación de los platos se fijó en $25 \mathrm{rpm}$ por ser la velocidad de giro de la paila durante el proceso de aplicación de los recubrimientos. La viscosidad se ensayó a 30 , 40 y $50{ }^{\circ} \mathrm{C}$ durante $60 \mathrm{~s}$ y las determinaciones se realizaron por triplicado.

Coeficiente de transmisión de vapor de agua Permeabilidad al vapor de agua

La permeabilidad al vapor de agua se determinó siguiendo el método ASTM E-96-80 (Gennadios, A., Weller, C. y Gooding, C., 1994), con modificaciones, sobre láminas constituidas a partir de las soluciones filmogénicas. Las mezclas, a $50 \pm 2{ }^{\circ} \mathrm{C}$, se extendieron sobre una superficie anti-adherente para lograr una lámina de $2,5 \mathrm{~mm}$ de espesor, se dejaron secar y se cortaron piezas cuadradas de $5 \mathrm{~cm}$ de lado. Brevemente, el índice de movimiento de vapor de agua, se cuantificó por gravimetría mediante las variaciones en el peso de sílica gel, con $0 \%$ de humedad inicial, confinada en una celda de permeación (Gennadios et al., 1994). Se emplearon soluciones saturadas de $\mathrm{KCl}, \mathrm{CaCl}_{2}$, o $\mathrm{KOH}$ que generan 90,37 y $10 \%$ de humedad relativa (HR) respectivamente a $23{ }^{\circ} \mathrm{C}$ (Jiménez Muñoz y Martínez Rodríguez, 1996) (Tabla 2). La humedad relativa, presión relativa y temperatura se controlaron mediante sensores PASCO modelo PS2114 (Estados Unidos, 2001).

La HR dentro de las celdas fue siempre menor que en el exterior y la diferencia de presión parcial

Tabla 2. Soluciones salinas saturadas y Humedad Relativa (HR) generada a $23^{\circ} \mathrm{C}$

\begin{tabular}{lc}
\hline \multicolumn{2}{c}{$\operatorname{Temp}^{2} 23^{\circ} \mathrm{C} \pm 0,5^{\circ} \mathrm{C}$} \\
\hline Solución saturada & $\mathrm{HR}(\%)$ \\
\hline $\mathrm{KCl}$ & 90 \\
$\mathrm{CaCl} 2$ & 37 \\
$\mathrm{KOH}$ & 10 \\
\hline
\end{tabular}

de vapor de agua a ambos lados de la lámina de biopolímero proporcionó la fuerza impulsora para el flujo de vapor agua a través de ella. El aumento de peso de la sílica en la celda se monitoreó a lo largo del tiempo, y se obtuvo una relación lineal. La pendiente de la recta se calculó por regresión y el coeficiente de transmisión de vapor de agua CTVA (g. $\mathrm{m}^{-2} \cdot \mathrm{h}^{-1}$ ) se calculó usando la Ecuación 1:

$$
\mathrm{CTVA}=\frac{\Delta \mathrm{m}}{(\mathrm{A} \cdot \Delta \mathrm{t})}
$$

Donde $\Delta m$ es la masa de agua ganada $(\mathrm{g}), A$ es el área expuesta de la lámina $\left(\mathrm{m}^{2}\right)$ y $\Delta t$ es el tiempo (h).

La propiedad de barrera, determinada como permeabilidad al vapor de agua, PVA $\left(\mathrm{g} \cdot \mathrm{m}^{-1} \cdot \mathrm{h}^{-1} \cdot \mathrm{Pa}^{-1}\right)$ se calculó según la Ecuación 2:

$$
P V A=\frac{C T V A \cdot x}{(\Delta P)}
$$

Donde $x$ es el espesor de la lámina de biopolímero $(\mathrm{m})$ y $\Delta P$ es la diferencia de presión de vapor de agua a través de la lámina $(\mathrm{Pa})($ Martucci y Ruseckaite, 2010). Los ensayos de permeabilidad se realizaron por duplicado para cada valor de HR, y el peso de la sílica se registró a las 2, 4 y 24 h.

\section{Solubilidad en agua}

El material soluble total de los biopolímeros se determinó según Martucci y Ruseckaite (2010), con modificaciones. Se empleó una porción de biopelícula, secada hasta peso constante $(\mathrm{m0})$. La $\mathrm{m0}$ se sumergió en $30 \mathrm{~mL}$ de agua destilada y se mantuvo en estufa a $30^{\circ} \mathrm{C}$ durante $24 \mathrm{~h}$. La porción de lámina de biopolímero no solubilizada en agua se separó por centrifugación durante 5 min a 1100 xg y posteriormente se secó en estufa a $105^{\circ} \mathrm{C}$ hasta peso constante (mf). El material solubilizado (MS) se calculó según la Ecuación 3:

$$
\mathrm{MS}=\frac{(\mathrm{m0}-\mathrm{mf})}{\mathrm{m0}} \times 100
$$

\section{Aplicación de recubrimiento biopolimérico a semillas}

Los recubrimientos a semillas maní, en lotes de $1,5 \mathrm{~kg}$, se aplicaron en paila rotatoria. Se adicionaron por goteo, $60 \mathrm{~mL}$ del biopolímero por $\mathrm{kg}$ de semillas a temperatura ambiente $\mathrm{o}$ 
$50{ }^{\circ} \mathrm{C}$. El goteo de las mezclas se realizó con la paila girando a $25 \mathrm{rpm}$ y el equipo se mantuvo funcionando durante $10 \mathrm{~min}$ hasta verificar la aparente homogeneidad en la distribución del color sobre la superficie en la masa de semillas.

Las semillas recubiertas se secaron aplicando convección forzada de aire a $93 \mathrm{~m}^{3} / \mathrm{h}$ a $25^{\circ} \mathrm{C}$ con la paila en funcionamiento o se depositaron sobre un papel absorbente y se mantuvieron a $25{ }^{\circ} \mathrm{C}$ hasta verificar el secado al tacto. Los ensayos se realizaron por duplicado.

\section{Caracterización de las semillas recubiertas}

\section{Espesor de capa}

El tegumento de las semillas recubiertas y no recubiertas fue separado de las semillas y el espesor de la lámina se determinó con un micrómetro mecánico marca Schwyz, capacidad 0-1mm, división mínima 1 um (Suecia).

\section{Humedad en semillas}

El contenido de humedad de semillas recubiertas se evaluó siguiendo la norma internacional 934.01 de la Association of Official Analytical Collaboration International (AOAC, 2010) con modificaciones. Brevemente se registró el peso inicial de un lote de semillas y posteriormente se las mantuvo en estufa con circulación forzada a $105^{\circ} \mathrm{C}$ hasta peso constante. Los ensayos se realizaron por triplicado.

Permeabilidad al vapor de agua en semillas recubiertas

La capacidad de absorción de agua de biopolímeros aplicados a semillas se ensayó colocando un lote de $5 \mathrm{~g}$ de semillas recubiertas en un desecador de $5 \mathrm{~L}$ de volumen, dentro del cual se colocó debajo del disco basal, una solución saturada de $\mathrm{KCl}$ que genera $90 \%$ de $\mathrm{HR}$ a $23{ }^{\circ} \mathrm{C}$ (Jiménez Muñoz y Martínez Rodríguez, 1996) (Tabla 2). El recipiente cerrado se mantuvo a $23 \pm 0,5^{\circ} \mathrm{C}$ en estufa y las semillas se pesaron a las 24,72 y $96 \mathrm{~h}$. El contenido de humedad del recipiente se registró colocando en su interior una sonda PASCO modelo PS-2114 (Estados Unidos, 2001). Las determinaciones se realizaron por triplicado.

\section{Resistencia mecánica y fluidez}

Los ensayos de resistencia mecánica en semillas recubiertas se realizaron usando un disco de siembra de placa inclinada de 26 alvéolos. La velocidad de rotación se ajustó a 27,5 rpm, asumiendo una velocidad de sembradora de 6,5 $\mathrm{km} \cdot \mathrm{h}^{-1}$. Dependiendo del calibre de las semillas, cada alvéolo puede albergar de dos a cuatro semillas a fin de liberar 18 semillas por metro lineal. Para posibilitar el pasaje de $500 \mathrm{~g}$ de semillas calibre 6-7, el disco giró 37 veces. La resistencia al daño mecánico se evaluó haciendo discurrir la misma muestra de semillas tres veces por el disco y registrando el porcentaje peso en peso $(\% \mathrm{p} / \mathrm{p})$ de las semillas dañadas colectadas en tres giros de disco. En el análisis, se asumió que las semillas deterioradas son el producto del choque y corte dentro de la placa de siembra al ingresar a los alvéolos. La repetición del procedimiento tuvo por objeto emular algunas de las operaciones comunes como clasificación, embolsado, apilado y carga en la tolva para siembra; todas maniobras que promueven daños en las semillas por aumento de la fricción. Los ensayos se realizaron por triplicado.

Para la determinación de la fluidez, se registró, en tres muestras independientes, el peso $(\mathrm{g})$ de las semillas descargadas en tres giros del disco. Del mismo modo que para determinar el daño, el procedimiento se repitió haciendo discurrir tres veces la misma muestra por el sistema (pasadas). Los ensayos se realizaron por triplicado.

\section{Angulo de reposo}

El ángulo de reposo es un parámetro adicional de fluidez y se define como el ángulo formado por la generatriz y la base de un cono de material particulado. A mayor fluidez, menor es el ángulo de reposo (Rojas Barahona y Aristizábal Torres, 2011). Para la determinación del ángulo de reposo se colocó una muestra de $1,5 \mathrm{~kg}$ de semillas en un embudo, la boca de salida de $40 \mathrm{~mm}$ de diámetro se apoyó en una superficie horizontal y el embudo se elevó en forma vertical hasta una altura de $50 \mathrm{~cm}$ hasta su vaciado. Se midió el ángulo de reposo del cono formado por las semillas con un goniómetro de precisión.

\section{Análisis estadístico}

Los resultados fueron expresados como las medias aritméticas $( \pm)$ el error estándar. Las diferencias de medias fueron comparadas usando el test DGC con el software InfoStat (Di Rienzo et al., 2008).

\section{RESULTADOS Y DISCUSION}

En este trabajo se ensayó la formulación de seis biopolímeros de recubrimiento para semillas de maní, empleando componentes orgánicos y 
biodegradables, de bajo costo y alta disponibilidad en el mercado. Cuatro formulaciones se realizaron a base de almidón de maíz (R_1 a R_4) y dos a base de almidón de mandioca (R_5 y R_6); como componentes menores para ambos almidones se utilizaron colágeno parcialmente hidrolizado y glicerol.

\section{Composición de biopolímeros y temperaturas de aplicación y secado}

El almidón del maíz usualmente contiene $70-80 \%$ de amilopectina y $20-30 \%$. de amilosa y la fracción no glucosídica se compone de 0,1 a 0,7 \% de proteínas y $1,5 \%$ de lípidos, principalmente ácidos grasos y lisofosfolípidos (Pérez y Bertoft, 2010). En la formación de geles de almidón de maíz, se ha reportado que la gelatinización depende de la velocidad de calentamiento (Pineda Gómez, Coral, Arciniegas, Rorales Rivera y Rodríguez García, 2010) y para almidones ricos en amilosa, se citan temperaturas de gelatinización entre $80^{\circ} \mathrm{C}$ (Badui Dergal, 1990) y $95{ }^{\circ} \mathrm{C}$ (Peressini, Bravin, Lapasin, Rizzotti y Sensidoni, 2003). En este trabajo, considerando que el grado alimenticio del almidón empleado no permitía conocer la relación amilosa:amilopectina, se seleccionó $90^{\circ} \mathrm{C}$ como temperatura de gelatinización para las formulaciones que contuvieron almidón de maíz, teniendo en cuenta además que los almidones de cereales poseen entalpías más altas que los obtenidos a partir de tubérculos o raíces (López y García, 2012).

La formulación R_1 se preparó con $10 \%$ de almidón de maíz, la R_2 y R_4 con 5 \% y la R_3 con $3 \%$ de almidón de maíz. La R_4 se formuló con menor contenido tanto de colágeno como de glicerol (Tabla 1). La mezcla R_1 formó una biopelícula gruesa, irregular y carente de homogeneidad sobre semillas cuando la operación de recubrimiento se realizó tanto a $25^{\circ} \mathrm{C}$ como a $50^{\circ} \mathrm{C}$ (no mostrado). En las formulaciones R_2 y R_4, con $5 \%$ de almidón, la aplicación a $50{ }^{\circ} \mathrm{C}$ redujo la viscosidad y mejoró la distribución, pero resultó de gran espesor y por los tanto de escasa aptitud para el uso (no mostrado). La homogeneidad en la distribución mejoró con la formulación tres R_3 aplicada a $50{ }^{\circ} \mathrm{C}$ (Figura $1 \mathrm{~A}$ $y E)$.

A diferencia de otros almidones de importancia comercial, el almidón de mandioca se caracteriza por poseer escasos niveles de residuos como grasas, proteínas y cenizas. La baja proporción de fracciones no glucosídicas y el contenido de proteínas y lípidos inferiores al 0,2 \%, aspectos que claramente lo diferencian de los almidones provenientes de cereales, impiden la formación de complejos entre amilosa y lípidos (Wheatley, Chuzel y Zakhia, 2003). En el almidón de mandioca se han reportado contenidos de amilosa de alrededor de 16-20\%, muy inferiores a otros almidones, y tanto la amilosa como la amilopectina que lo componen poseen alto peso molecular (Breuninger, Piyachomkwan y Sriroth, 2009).

La gelatinización del almidón de mandioca tiene lugar a temperaturas relativamente bajas, con valores iniciales de $60^{\circ} \mathrm{C}$ y finales de $80^{\circ} \mathrm{C}$. En este trabajo se seleccionó $70^{\circ} \mathrm{C}$ como la temperatura de gelatinización para las formulaciones R_5 y R_6, las cuales contenían $3 \%$ y $5 \%$ de almidón de mandioca respectivamente.

En la gelatinización, a medida que incrementa la temperatura, el gránulo de almidón de mandioca aumenta de diámetro y en el punto de máxima viscosidad, sus capas externas se desestructuran y posibilitan la continua absorción de agua. Durante el enfriamiento se produce un aumento de la viscosidad que luego desciende y se estabiliza. Con el descenso de la temperatura, se observa cierta pérdida de claridad y trasparencia, que es máxima, a la temperatura de gelatinización (Breuninger et al., 2009). En las mezclas ensayadas en este trabajo, se observó que los geles de mandioca fueron siempre más translúcidos que los formulados a base de almidón de maíz (no mostrado).

La baja tendencia a la retrogradación del almidón de mandioca, que posibilita la formación de un gel claro, elástico y estable, puede asociarse a la menor composición relativa de amilosa respecto del almidón de maíz; dado que la amilosa tiene una alta tendencia a retrogradarse y producir geles resistentes y películas fuertes (Pérez y Bertoft, 2010). El comportamiento en la cobertura de semillas de las formulaciones a base de almidón de mandioca mostró que las mezclas con $3 \%$ y $5 \%$, R_5 y R_6 respectivamente, formaron una película homogénea sobre las semillas, tanto cuando fueron aplicadas a $50{ }^{\circ} \mathrm{C}$ como a temperatura ambiente, lo que representó una ventaja a favor de estas formulaciones respecto de las que contienen almidón de maíz (Figura $1 \mathrm{D}$ y F).

El tiempo de secado de las biopelículas aplicadas sobre semillas, se determinó sin y con aporte de circulación forzada de aire a $93 \mathrm{~m}^{3} \cdot \mathrm{h}^{-1} \mathrm{y}$ $25^{\circ} \mathrm{C}$. El tiempo promedio de secado sin circulación forzada de aire de las semillas recubiertas con biopolímeros a base de almidón de maíz y mandioca fue de 45 min y 30 min respectivamente. Estos valores se redujeron a 5 min cuando se 


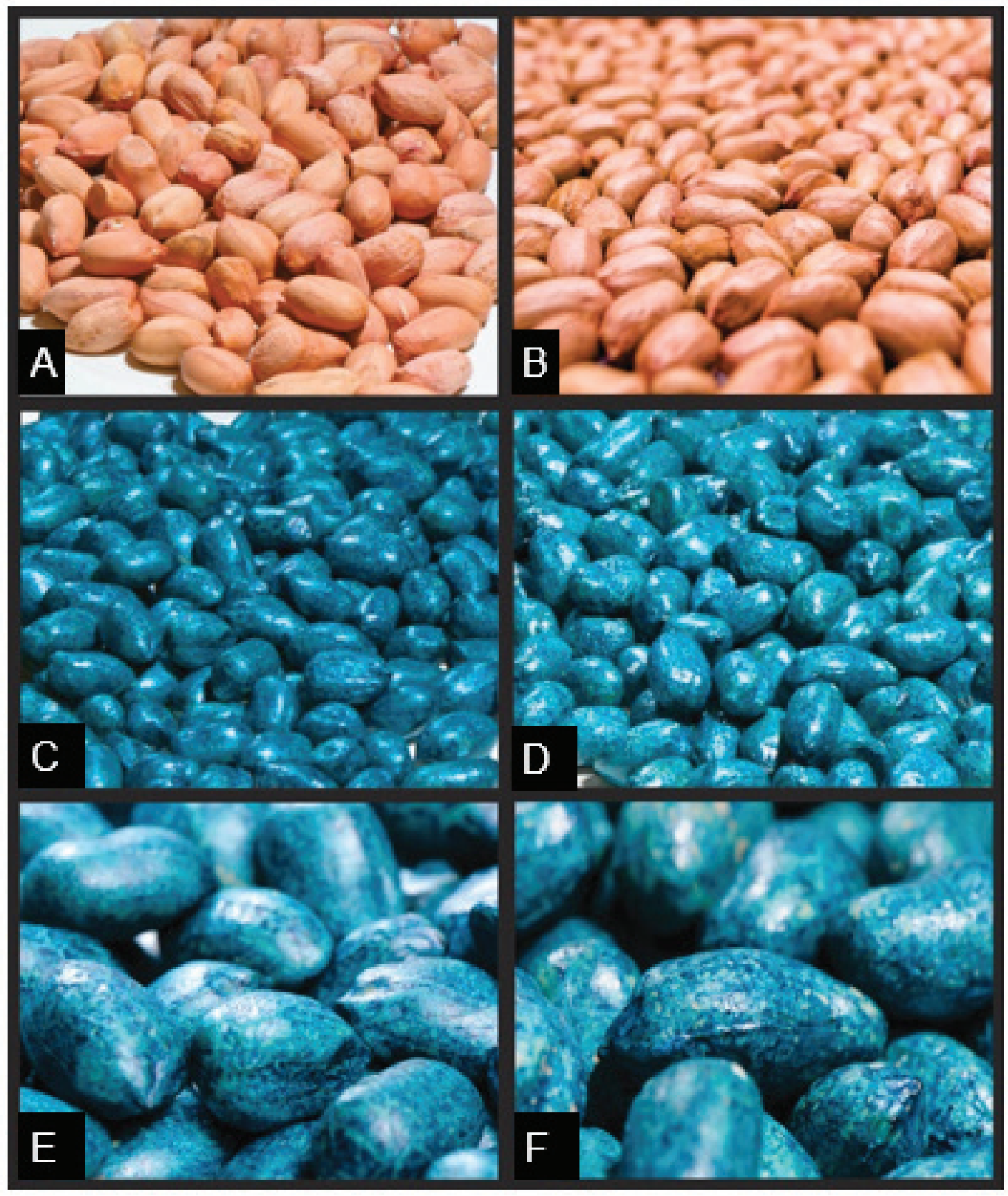

Figura 1. Semillas de maní Cv. Granoleico recubiertas con $60 \mathrm{~mL} . \mathrm{kg}^{-1}$ de biopolímeros. A. No recubiertas, B. Polímero Becker Underwood®, C. Biopolímero R_3 (3 \% de almidón de maíz), D. Biopolímero R 5 (3 \% de almidón de mandioca), E. Macro con recubrimiento R_3, F. Macro con recubrimiento R_5.

aplicó aire por convección forzada, sin diferencias respecto de los almidones constituyentes de las biopelículas (no mostrado).

Considerando el espesor de capa y la homogeneidad en la distribución superficial de las biopelículas analizadas, resultaron, tecnológicamente aptas para la aplicación en semillas, las formulaciones R_3 y R_5. Por tanto, los análisis físicos de los biopolímeros se describen sobre estas formulaciones. 


\section{Caracterización de los biopolímeros}

\section{Viscosidad}

Conocer la reología de los biopolímeros aporta información de su comportamiento durante la dispersión en la constitución de la mezcla y aplicación de la cobertura a las semillas y permite además definir las condiciones operativas en los equipos de transporte e inyección de los fluidos para considerar el escalado de la formulación y aplicación en volúmenes mayores (Steffe, 1996).

La viscosidad se determinó con un reómetro en los biopolímeros R_3 y R_5 durante $60 \mathrm{~s}$ a 30, 40 y $50{ }^{\circ} \mathrm{C}$, aplicando fuerzas con una geometría de platos paralelos.

En ambas mezclas y en todas las temperaturas ensayadas la viscosidad se mantuvo constante a lo largo del tiempo (Figura $2 \mathrm{~A}$ ).

La interacción temperatura ${ }^{*}$ viscosidad mostró diferencias significativas $(p>0,05)$ en ambas mezclas y en todas las combinaciones (Tabla 3). La menor viscosidad, se observó en el biopolímero de mandioca R_5 a las temperaturas más altas y esta misma formulación mostró un valor de viscosidad significativamente mayor a $30^{\circ} \mathrm{C}$ (Tabla 3 ).

La mayor viscosidad aparente de la mezcla filmógena de almidón de mandioca podría relacionarse con su mayor contenido de amilopectina, que contribuye a reforzar la red de amilosa. Las características estructurales de sus macromoléculas, como pesos moleculares, grado de polimerización, distribución y tamaño de sus cadenas refuerzan la estructura de la red (López y García, 2012).

En ambas mezclas el aumento de la temperatura redujo la viscosidad (cP). Para R_3 a base de almidón de maíz, se observó que la viscosidad se redujo de modo lineal $\left(R^{2}=85,03\right)$ con el incremento de la temperatura, según la Ecuación 4: $\mathrm{Cp}=329,2-2,17^{\star} \mathrm{T}$ (Figura $2 \mathrm{~B}$ ). Para la mezcla R_5 el comportamiento ajustó a un modelo doble inverso, $\left(R^{2}=97,06\right)$ Ecuación 5: $\mathrm{Cp}=1 / 0,0127-0,30 /$ T (Figura $2 \mathrm{C}$ ).

Coeficiente de transmisión de vapor de agua Permeabilidad al vapor de agua en láminas de biopolímero

El movimiento de vapor de agua a través de una lámina de biopolímero, permite conocer y evaluar el funcionamiento de estas biopelículas como barrera y predecir el grado de preservación que conferirán a la semilla en el recubrimiento (Gennadios et al., 1994).

El coeficiente de transmisión de vapor de agua

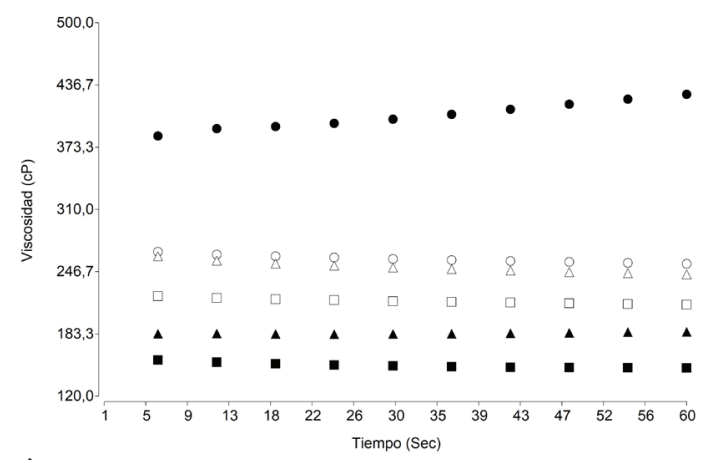

A

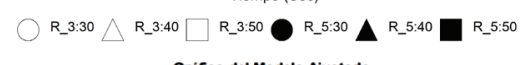

Gráfico del Modelo Aiustado

Viscosidad maiz $(C P)=329,22-2,173^{\circ}$ Temperatura $\left({ }^{\circ} \mathrm{C}\right)$

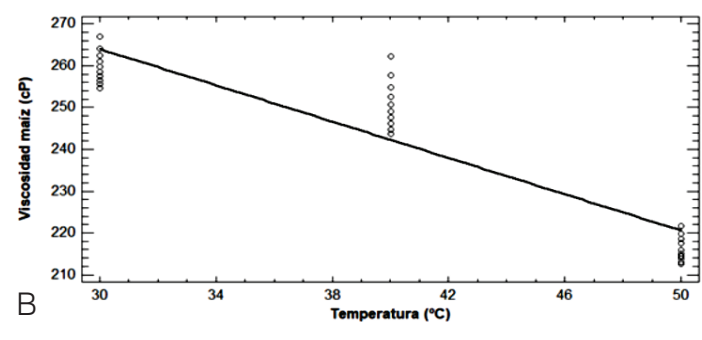

Gráfico del Modelo Ajustado

Viscosidad mandioca $(\mathrm{cP})=1 /\left(0,0127749-0,30152 /\right.$ Temperatura $\left.\left({ }^{\circ} \mathrm{C}\right)\right)$

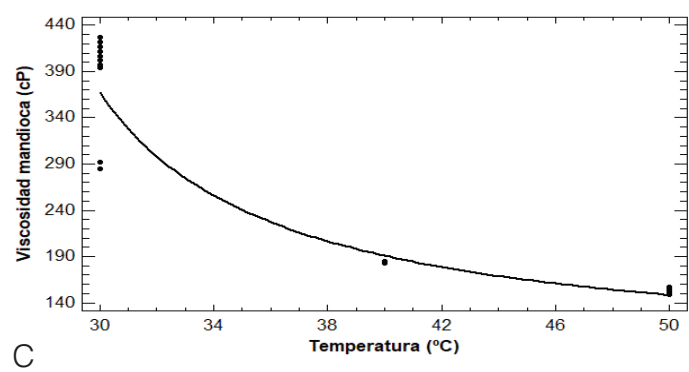

Figura 2. Viscosidad (cP) de biopolímeros de recubrimiento R_3 (3\% almidón de maíz) y R_5 (3\% almidón de mandioca) a 30, 40 y $50^{\circ} \mathrm{C}$. Variación en función del tiempo (A) y Regresiones en función de la temperatura en R_3 (B) y R_5 (C).

Tabla 3. Viscosidad ( $\mathrm{CP}$ ) de biopolímeros de recubrimiento con 3 \% almidón de maíz (R_3) y 3 \% almidón de mandioca (R_5) a 30,40 y $50^{\circ} \mathrm{C}$

\begin{tabular}{lccc}
\hline Recubrimiento & Temp $^{\circ} \mathrm{C}$ & $\mathrm{CP}$ & \\
\hline R_5 & 50 & $144,39 \pm 3,27$ & A \\
R_5 & 40 & $185,51 \pm 3,27$ & B \\
R_3 & 50 & $213,74 \pm 3,27$ & C \\
R_3 & 40 & $244,75 \pm 3,27$ & D \\
R_3 & 30 & $255,22 \pm 3,27$ & E \\
R_5 & 30 & $427,78 \pm 3,27$ & F \\
\hline
\end{tabular}

Medias con una letra común no son significativamente diferentes DGC $(p>0,05)$.

(CTVA) se ensayó en los biopolímeros de almidón de maíz R_3 y mandioca R_5 a 10, 37 y 90 \% de HR y para ambas biopelículas; los mayores valores de 
CTVA se observaron con la HR más alta (Tabla 4). Se observaron diferencias significativas en el coeficiente de transmisión de vapor de agua a través de las biopelículas en los tres niveles de HR ensayados. En $37 \%$ de HR, la interacción biopolímero*HR mostró que las láminas a base de almidón de maíz R_3 y mandioca R_5 difirieron significativamente $(p>0,05)$ en el CTVA mientras que la incorporación de agua en $10 \%$ y $90 \%$ de HR no mostró diferencias entre las biopelículas. A $37 \%$ de HR se observó que, a través de la lámina a base de almidón de mandioca, el CTVA fue $57 \%$ mayor que a través de la lámina a base de almidón de maíz (Tabla 4).

La permeabilidad al vapor de agua (PVA) de una película es una constante, independiente de la diferencia de presión de vapor $(\Delta P)$ que genera la fuerza motriz entre el interior y el exterior de la celda de permeación en una atmósfera de HR controlada. Es decir que, en una película sometida a diferentes gradientes de presión de vapor, el flujo de vapor de agua a través de ella difiere, pero su permeabilidad calculada (PVA) es constante si no hay variaciones de temperatura. Sin embargo, esto no ocurre en las películas comestibles hidrófilas, debido a que las moléculas de agua interactúan con los grupos polares en la estructura de la película causando plastificado o hinchazón (Hema Prabha y Vasudevan Ranganathan, 2018). Los resultados obtenidos demostraron que la permeabilidad al vapor de agua no varió entre las biopelículas a las HR más bajas y fue significativamente más alta en la constituida con almidón de mandioca (R_5) a 90 \% HR (Tabla 4). Dado que la PVA calculada es un valor obtenido a partir de datos de gravimetría del sistema constituido por la cámara de permeación junto a la biopelícula, es posible considerar que los incrementos observados a $90 \%$ de HR puedan deberse, no sólo a la permeabilidad al vapor de agua, sino también a interacciones de las moléculas de agua con los constituyentes de la biopelícula. Esto se funda en la naturaleza hidrófila tanto de los almidones como del glicerol empleado como plastificante, aun cuando el colágeno parcialmente hidrolizado tiene como función actuar de barrera efectiva al vapor de agua (Bourtoom, 2008). En este sentido, han sido reportadas variaciones en PVA calculados en otros tipos de películas alimenticias celulósicas y a base de amilosa (Rankin, Wolff, Davis y Rist, 1958; Woodruff, Peck y Banker, 1972). La hidrofilia de los almidones se asocia a que poseen tres grupos hidroxilo por unidad de D-glucósido, y su contenido de humedad en equilibrio es entre 10 y $20 \%$. Debido a que el agua es un plastificante natural, las pérdidas o ganancias de humedad pueden resultar en variaciones significativas en las propiedades de barrera (Willett, 2009).

Los mayores valores de PVA observados a $90 \%$ de HR en la mezcla R_5, pueden asociarse a las mayores ramificaciones en la estructura debido al contenido de amilopectina en el almidón de mandioca. Esta conformación molecular reduce la capacidad de constituir entrecruzamientos estrechos y por tanto resultan con mayor permeabilidad al vapor de agua (Willett, 2009). Contrariamente, valores más altos en las propiedades de barrera, se observan en mezclas que posee almidones con mayor proporción de amilosa, debido a que esta red presenta una estructura con fuerte ordenamiento molecular, formando películas más densas como ocurre en las biopelículas que contienen almidón de maíz (Hema Prabha y Vasudevan Ranganathan, 2018). No obstante, este tipo de redes tiene un comportamiento menos elástico y con mayor tendencia a resquebrajarse cuando ocurren pérdidas importantes de humedad (Willett, 2009).

\section{Solubilidad en agua}

La solubilidad de los biopolímeros se analizó sumergiendo las muestras en agua a $30{ }^{\circ} \mathrm{C}$. Sin mostrar diferencias significativas en la respuesta, el $42,3 \pm 1,27 \%$ de la muestra a base de almidón de maíz (R_3) y el 36,01 $\pm 1,57 \%$ de la muestra a base de almidón de mandioca (R_5), permaneció íntegro luego de $24 \mathrm{~h}$. Estos resultados coincidieron con otros investigadores que propusieron que el tipo

Tabla 4. Coeficiente de transmisión de vapor de agua, CTVA $\left(\mathrm{g} \mathrm{m}^{-2} \mathrm{~h}^{-1}\right)$ y permeabilidad al vapor de agua, $\mathrm{PVA}\left(\mathrm{g} \mathrm{m}^{-1} \mathrm{~h}^{-1} \mathrm{~Pa} \mathrm{a}^{-1}\right)$ en biopolímeros formulados con almidón de maíz (R_3) y mandioca (R_5) en atmósferas de humedad relativa (HR) controladas

\begin{tabular}{lcccccc}
\hline Recubrimiento & HR $(\%)$ & \multicolumn{2}{c}{ CTVA $\left(\mathrm{g} \mathrm{m}^{-2} \mathrm{~h}^{-1}\right)$} & \multicolumn{3}{c}{ PVA $\left(\mathrm{g} \mathrm{m}^{-1} \mathrm{~h}^{-1} \mathrm{~Pa}^{-1}\right)$} \\
\hline R_3 & 10 & $1,26 \pm 0,29$ & A & $6,57 \mathrm{E}-07$ & $\pm 1,47$ E-07 & A \\
R_5 & 10 & $1,26 \pm 0,30$ & A & 8,11 E-07 & $\pm 1,85$ E-07 & A \\
R_3 & 37 & $3,86 \pm 0,29$ & B & 5,70 E-07 & $\pm 4,02$ E-08 & A \\
R_5 & 37 & $6,71 \pm 0,30$ & C & 4,62 E-07 & $\pm 2,00$ E-08 & A \\
R_3 & 90 & $32,98 \pm 0,30$ & D & 23,00 E-07 & $\pm 1,65$ E-08 & B \\
R_5 & 90 & $33,31 \pm 0,29$ & D & 46,40 E-07 & $\pm 2,09$ E-08 & C \\
\hline
\end{tabular}

Medias con una letra común no son significativamente diferentes DGC $(p>0,05)$. 
de almidón empleado en las biopelículas no incide en la solubilidad en agua, independientemente de la temperatura a la que se los ensaya (López y García, 2012).

\section{Aplicación de recubrimiento biopolimérico a semillas}

Con las formulaciones R_3 y R_5, fue posible conseguir un recubrimiento uniforme de las semillas de maní empleando la paila (Figura $1 \mathrm{E} \mathrm{y}$ F). Cuando las mezclas se aplicaron a $50^{\circ} \mathrm{C}$ como una operación tendiente a reducir la viscosidad, las biopelículas se distribuyeron homogéneamente sobre las semillas. La necesidad de reducción de viscosidad aumentando la temperatura se verificó en la mezcla R_3, donde a su vez la aplicación a temperatura ambiente dio como resultado una película heterogénea. Por su parte, la mezcla R_5 se distribuyó de modo uniforme a ambas temperaturas. El comportamiento de la película de estos biopolímeros sobre las semillas, se analizó de modo comparativo con la formulación comercial del polímero Becker Underwood $\circledast$ (Figura 1 B). Se evaluaron parámetros de conservación, preservación de la integridad, resistencia a daño mecánico y fluidez en maquinaria de siembra.

\section{Caracterización de las semillas recubiertas}

Espesor de capa y contenido de humedad en semillas recubiertas

El espesor de la lámina de las biopelículas de recubrimiento se determinó desprendiendo el tegumento de las semillas y evaluándolo con micrómetro. Las diferencias fueron significativas $(p>0,01)$ respecto de las semillas no recubiertas, pero sin diferencias entre los biopolímeros y la fórmula comercial. Se observó que el aporte de los recubrimientos al espesor del tegumento fue, en promedio, de $10 \mu \mathrm{m}$ (Tabla 5).

Tabla 5. Espesor de capa y Humedad en semillas No recubiertas (No_R) y recubiertas con biopolímero formulados con almidón de maíz (R_3) y mandioca (R_5) y polímero comercial (Becker Underwood $($ )

\begin{tabular}{lcl}
\hline Recubrimiento & Espesor capa $(\mu \mathrm{m})$ & Humedad $(\%)$ \\
\hline No_R & $104,33 \pm 1,65 \mathrm{~A}$ & $5,65 \pm 0,12 \mathrm{~A}$ \\
R_Com & $112,00 \pm 1,66 \mathrm{~B}$ & $6,00 \pm 0,12 \mathrm{~A}$ \\
R_3 & $118,00 \pm 1,67 \mathrm{~B}$ & $6,84 \pm 0,12 \mathrm{~B}$ \\
R_5 & $110,67 \pm 1,68 \mathrm{~B}$ & $7,32 \pm 0,12 \mathrm{C}$ \\
\hline
\end{tabular}

Medias con una letra común no son significativamente diferentes DGC $(p>0,01)$

\section{Humedad de las semillas}

El contenido de humedad de las semillas recubiertas se analizó luego de su secado hasta peso constante; para todos los tratamientos el porcentaje de humedad final fue inferior a $7,5 \%$ (Tabla 5). En las semillas no recubiertas y en las recubiertas con el polímero comercial no se observaron diferencias significativas $(p>0,01)$, mientras que en las recubiertas con los biopolímeros R_3 y R_5 el contenido de humedad fue significativamente mayor y sin diferencias entre las formulaciones. El incremento en el contenido de humedad de las semillas recubiertas con la biopelícula R_3, conformada por almidón de maíz, fue del $21 \%$ respecto de las semillas sin recubrir. Para el caso de las semillas recubiertas con la biopelícula formulada con almidón de mandioca, el aumento en la humedad de las semillas fue 29,5 \% con relación a las no recubiertas (Tabla 5). El contenido de agua que cada fórmula retiene, se relaciona con las proporciones de amilosa:amilopectina de cada tipo de almidón y las estructuras de redes que conforman, más laxas e hidrofílicas en caso de almidón de mandioca y más compacta y ordenadas, y consecuentemente algo menos hidrófilas en caso de almidón de maíz (Willett, 2009).

\section{Permeabilidad al vapor de agua}

La propiedad de barrera de las biopelículas, evaluada a través de la incorporación de agua en las semillas recubiertas, se ensayó a lo largo de 96 h en una atmósfera de 90 \% HR. En estas condiciones de alta presión de vapor todas las muestras incorporaron agua (Figura 3). Hasta las $24 \mathrm{~h}$ y en términos relativos respecto del tiempo cero (T0), la interacción de las variables tiempo*recubrimiento mostró los menores

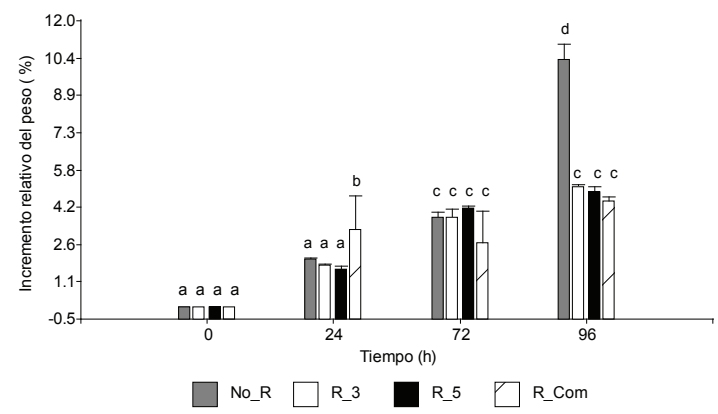

Figura 3. Permeabilidad al vapor de agua en biopolímeros sobre semillas. Incremento porcentual (\%) de peso relativo a TO. No recubiertas (No_R) y recubiertas con biopolímeros formulados con almidón de maíz (R_3) y mandioca (R_5) y polímero comercial (Becker Underwood®). Medias con una letra común no son significativamente diferentes DGC $(p>0,01)$ 
valores de incorporación de agua en semillas no recubiertas (No_R) y recubiertas con biopolímero de almidón de maíz (R_3) y mandioca (R_5), sin diferencias entre ellos $(p>0,01)$. Desde las $72 h$ y hasta el final del ensayo, el incremento en peso de las semillas fue significativo respecto al tiempo anterior, y no mostró diferencias entre R_3, R_5 y polímero comercial. En las semillas no recubiertas el aumento de peso por incorporación de agua, fue significativamente superior a las $96 \mathrm{~h}(\mathrm{p}>0,01)$. La permeabilidad al vapor agua respecto al T0, evaluado como variación porcentual del peso al final del ensayo, fue de 4,48 \%, 4,86 \% y $5,03 \%$ para las semillas recubiertas con polímero comercial y biopolímeros R_5 y R_3 respectivamente, sin diferencias significativas entre ellos ( $p>0,01)$; mientras que fue del $10,37 \%$ en semillas no recubiertas, mostrando el importante rol de barrera de los recubrimientos ensayados en ambientes de alta HR (Figura 3).

\section{Resistencia mecánica y fluidez}

El uso de recubrimientos en semillas tiene como objetivo principal conservar la calidad e integridad de las semillas en los procesos de mecanizado, disminuyendo su deterioro durante la manipulación, transporte y dosificación en la siembra; dado que, en la actualidad, mayoritariamente persiste el empleo de maquinarias de distribución mecánica de placa inclinada para la siembra de maní (Bongiovanni et al., 2012).

El análisis de la variación en la resistencia debido a la presencia de recubrimientos, se realizó haciendo pasar una muestra de semillas a través de un disco de siembra de placa inclinada de 26 alvéolos. El pasaje de las muestras de semillas a través de disco, se realizó tres veces consecutivas y se determinó el deterioro mecánico, como el porcentaje en peso de las semillas dañadas.

La proporción de semillas dañadas se cuantificó en relación a las colectadas en cada pasada. Las semillas no recubiertas mostraron los valores significativamente más altos $(p>0,01)$ en la segunda y tercera pasada por el disco de siembra, respecto de las semillas recubiertas con el polímero a base de almidón de maíz R_3, mandioca R_5 y el recubrimiento comercial que no mostraron diferencias entre sí en ninguna de las tres rondas de pasaje por el disco de siembra (Figura 4 A).

En el análisis de fluidez, definido por el peso ( $\mathrm{g}$ ) de las semillas colectadas luego de pasar a través del disco de siembra, se observan diferencias significativas $(p>0,01)$ cuando se analizó la interacción recubrimiento*pasadas a través del disco. La fluidez de las semillas no recubiertas no
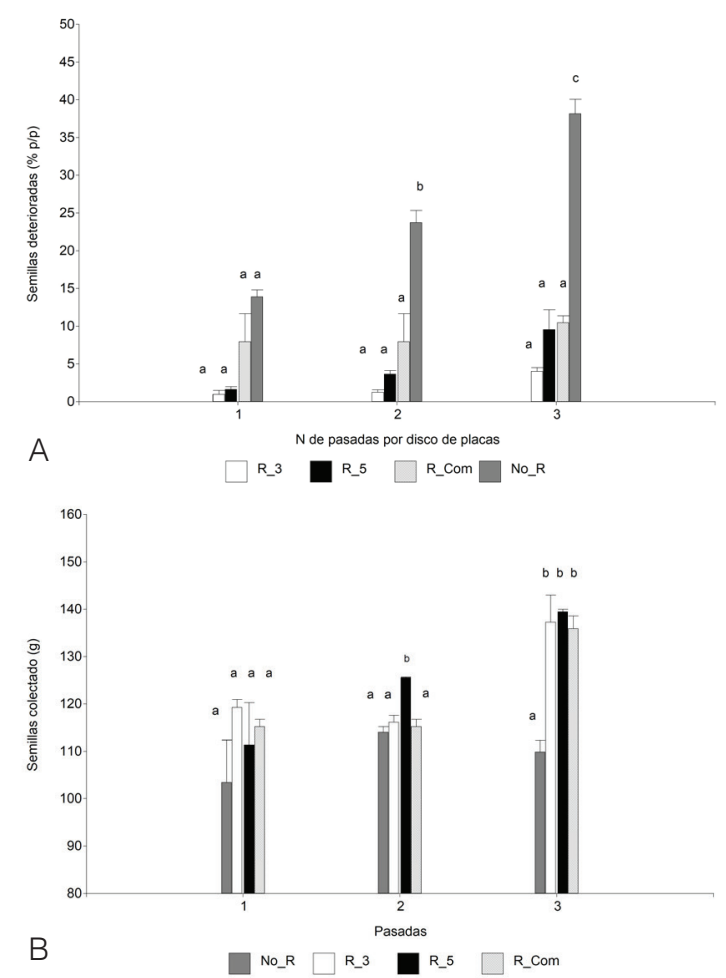

Figura 4. Comportamiento mecánico de semillas recubiertas en pasajes sucesivos (1-3) por discos de placa. A: Resistencia al daño mecánico. B: Fluidez semillas no recubiertas (No_R) y recubiertas con biopolímeros formulados con almidón de maíz (R_3) y mandioca (R_5) y polímero comercial (Becker Underwood(B). Medias con una letra común no son significativamente diferentes DGC $(p>0,01)$

varió con el número de pasadas y no se diferenció de las semillas recubiertas con biopolímeros R_3, R_5 y polímero comercial en la primera pasada. En la segunda pasada, las semillas recubiertas con el biopolímero a base de mandioca, (R_5) tuvieron mayor fluidez que el resto de los tratamientos. En la tercera pasada, en todas las muestras recubiertas, la fluidez fue significativamente mayor a las semillas no recubiertas. En términos relativos, la fluidez de las semillas recubiertas luego de tres pasadas fue 25,27 y $24 \%$ mayor en semillas recubiertas con R_3, R_5 y polímero comercial respecto de las semillas no recubiertas (Figura $4 \mathrm{~B}$ ).

Un procedimiento complementario para evaluar la fluidez en semillas, es determinar el ángulo de reposo, que se forma por la generatriz y la base de un cono del material. Este ángulo depende de variables como el tamaño, la forma, la densidad, la rugosidad, la adherencia y la humedad de las partículas analizadas. Cada tipo de semillas tiene definido su ángulo de reposo, que se encuentra íntimamente ligado a la fluidez, de modo que, 
a mayor fluidez, menor es el ángulo de reposo (Rojas Barahona y Aristizábal Torres, 2011). En coincidencia con los resultados de fluidez (Figura 4 B), el ángulo de reposo determinado para muestras de semillas no recubiertas (No_R) fue de $40^{\circ}$ mientras que para las semillas recubiertas con las biopelículas R_3, R_5 y el polímero comercial, los ángulos determinados con el goniómetro fueron de $35^{\circ}, 35^{\circ}$ y $31^{\circ}$ respectivamente, sin diferencias entre recubrimientos (no mostrado). Este conjunto de resultados indica que las formulaciones biopoliméricas ensayadas en este trabajo ofrecen igual protección frente al daño mecánico que el polímero comercial y adicionalmente el biopolímero R_5 formulado a base de almidón de mandioca mostró la mayor fluidez.

\section{CONCLUSIONES}

Este trabajo propuso constituir un biopolímero de recubrimiento adecuado a las características de las semillas de maní, utilizando componentes orgánicos, naturales de bajo costo y alta disponibilidad en el mercado.

Entre las formulaciones propuestas, los biopolímeros que contenían 3 \% de almidón de maíz (R_3) y mandioca (R_5) posibilitaron su correcta aplicación y distribución sobre las semillas en las condiciones ensayadas. Sin embargo, las características reológicas y otras propiedades físicas hacen elegible a la biopelícula a base de almidón de mandioca. En primer lugar, en esta mezcla la gelatinización ocurre a menor temperatura, lo que implica menor demanda energética durante su constitución. Si bien esta película mostró mayor viscosidad a la menor temperatura ensayada, esta condición no redujo su potencial en la aplicación sobre semillas a temperatura ambiente. Analizando el comportamiento no lineal de la viscosidad frente a la temperatura para el recubrimiento con almidón de mandioca, se observa que a temperaturas relativamente elevadas, su viscosidad es menor en comparación con el recubrimiento a base de almidón de maíz.

En cuanto a sus propiedades de barrera, analizando la permeabilidad al vapor de agua y coeficiente de transmisión de vapor en láminas, la formulación a base de almidón de mandioca mostró, al igual que la película constituida con almidón de maíz, similar comportamiento (en atmósferas de humedad relativa de $37 \%$ ), que si bien en los términos de los ensayos realizados en este trabajo representan los valores intermedios, esta condición de humedad supera las recomendadas para el almacenamiento de semillas y granos. Por lo que se considera que provee una barrera adecuada al vapor de agua.

La biopelícula a base de almidón de mandioca, formó una capa de similar espesor y permeabilidad al vapor de agua que la del polímero comercial con el que se la comparó. Asimismo, incrementó la resistencia al daño en ensayos de simulación de mecanización, a la vez que favoreció la fluidez en el mismo sentido que lo hizo la muestra comercial.

Este trabajo aporta una formulación para el recubrimiento biopolimérico de semillas de maní que, basado en componentes naturales de grado alimenticio y biodegradables, posee potencialidad de ser adoptado para el uso en el marco de la agricultura sustentable.

\section{AGRADECIMIENTOS}

Los autores agradecen a los alumnos Cintia Barbeito y Nicolás Caneto (Ingeniería Química Facultad de Ciencias Exactas, Físicas y Naturales de la Universidad Nacional de Córdoba) por su colaboración en algunos aspectos experimentales y al Dr. Pablo Gonzalez (Instituto Nacional de Tecnología Agropecuaria) por las fotografías.

Este trabajo fue financiado con fondos del convenio INTA AUDEAS CONADEV CIAC 94156. Res Cd.837-15 (2015-2018) y SeCyT-UNC Res. 313-2016 (2016-2017).

\section{BIBLIOGRAFÍA}

Association of Official Analytical Collaboration (AOAC) International (2010). Moisture in feed 934.01 Official Methods of Analysis of AOAC International - M (18 ed., $3^{\text {rd }}$ rev.). Rockville, EstadosUnidos.

Badui Dergal, S. (1990). Química de los Alimentos (2a ed.). México, DF., México: Alhambra Mexicana

Bolsa de Cereales de Córdoba (2019, Junio). Informe n 167-Primera quincena de junio 2019 Informe de cultivos - Departamento de Información Agroeconómica. Recuperado de http://www.bccba.com.ar/images db/noticias_archivos/4362Descargar\%20informe. pdf\#viewer.action=download

Bongiovanni, R, Troilo, L y Pedelini, R. (2012). Buenas Prácticas Agrícolas para la producción de maní. Córdoba, Argentina. Instituto Nacional de Tecnología Agropecuaria. Recuperado de: https://inta.gob. ar/sites/default/files/script-tmp-manual_-_buenas_ prcticas_agrcolas_para_la_produccin_d.pdf

Bourtoom, T. (2008). Review Article Edible films and coatings: characteristics and properties. International 
Food Research Journal, 15(3), 237-248.Recuperado de: http://ifrj.upm.edu.my/15\%20(3)\%202008/01.\%20 Bourtoom, \%20T.pdf

Breuninger, W., Piyachomkwan, K. y Sriroth, K. (2009). Tapioca/Cassava Starch: Production and Use. En J. N. BeMiller (Ed.), Starch: Chemistry and Technology (3a ed.) (541-568): Academic Press. DOl: https://doi. org/10.1016/B978-0-12-746275-2.00012-4

Secretaría de Agroindustria. Ministerio de Producción y Trabajo, República Argentina (2019). Cadena de mani - Resumen marzo 2019. Recuperado de: http:// www.alimentosargentinos.gob.ar/HomeAlimentos/ Cadenas\%20de\%20Valor\%20de\%20Alimentos\%20 y\%20Bebidas/informes/Resumen_Cadena_2019\%20 Mani_MARZO_2019.pdf

Di Rienzo, J. A., Casanoves, F., Balzarini, M. G., González, L., Tablada, M., Robledo, C. W. InfoStat (versión 2008) [Software]. Córdoba, Argentina: Grupo InfoStat, FCA, Universidad Nacional de Córdoba. URL: http://www. infostat.com.ar

Gennadios, A., Weller, C. y Gooding, C. (1994). Measurement Errors in Water Vapor Permeability of Highly Permeable, Hydrophilic Edible Films. Journal of Food Engineering, 21, 395-409. DOI: https://doi. org/10.1016/0260-8774(94)90062-0

Hema Prabha, P. y Vasudevan Ranganathan, T. (2018). Process optimization for evaluation of barrier properties of tapioca starch based biodegradable polymer film. International Journal of Biological Macromolecules, 120 (Part A), 361-370. DOI: https://doi.org/10.1016/j. ijbiomac.2018.08.100

Jiménez Muñoz, F. y Martínez Rodríguez, F. (1996). Efecto de la humedad relativa sobre la estabilidad física de algunos sistemas ternarios utilizados en el diseño de formas farmacéuticas liquidas de administración peroral. Revista colombiana de ciencias químicofarmacéuticas, 25, 60-70. Recuperado de: https:// revistas.unal.edu.co/index.php/rccquifa/article/ view/56468

López, O. y García, M. (2012). Starch films from a novel (Pachyrhizus ahipa) and conventional sources: Development and characterization. Materials Science and Engineering, C 32, 1931-1940. DOI: https://doi. org/10.1016/j.msec.2012.05.035

Martucci, J. y Ruseckaite, R. (2010). Biodegradable Bovine Gelatin/Na+-Montmorillonite Nanocomposite Films. Structure, barrier and dynamic mechanical properties. Journal Polymer-Plastics Technology and Engineering, 49, (6). 581-588. DOI: https://doi. org/10.1080/03602551003652730

Peressini, D., Bravin, B., Lapasin, R., Rizzotti, C. y Sensidoni, A. (2003). Starch-methylcellulose based edible films: rheological properties of film-forming dispersions. Journal of Food Engineering, 59, 25-32. DOI: https://doi.org/10.1016/S0260-8774(02)00426-0

Pérez, S. y Bertoft, E. (2010). The molecular structures of starch components and their contribution to the architecture of starch granules: A comprehensive review. Starch/Stärke, 62, 389-420. DOI: https://doi. org/10.1002/star.201000013

Pineda-Gómez, P., Coral, D., Arciniegas, M., RoralesRivera, A. y Rodríguez García, M. E. (2010). Papel del agua en la gelatinización del almidón de maíz: estudio por calorimetría diferencial de barrido. Ingeniería y Ciencia,6(11), 129-141. Recuperado de: http://www. scielo.org.co/pdf/ince/v6n11/v6n11a08.pdf.

Rankin, J., Wolff, I., Davis, H. y Rist, C. (1958). Permeability of Amylose Film to Moisture Vapor, Selected Organic Vapors, and the Common Gases. Industrial and Engineering Chemistry, 3(1) 120-123. DOI: https://doi. org/10.1021/i460003a023

Rojas Barahona, A. y Aristizábal Torres, I. (2011). Efecto del contenido de humedad sobre propiedades físicas de la semilla de vitabosa (Mucuna deeringiana). Revista de la Facultad Nacional de Agronomía de Medellín, 64 (1) 5961-5971. Recuperado de: http:// www.scielo.org.co/pdf/rfnam/v64n1/a19v64n01.pdf

Steffe, J. (1996). Rheological methods in food process engineering (2a ed.). East Lansing, Estados Unidos: Freeman Press.

Wheatley, C., Chuzel, G. y Zakhia, N. (2003). CASSAVA / The Nature of the Tuber. En B. Caballero, P. Finglas, F. Toldrá (Eds.), Encyclopedia of Food Sciences and Nutrition (964-969). Academic Press.

Willett, J. L. (2009). Starch in polymer compositions Ch 19 J. BeMiller and R. Whistler (Eds.) Starch Chemistry and Technology Food Science and Technology (715743) Estados Unidos. Elsevier Science. DOI: https:// doi.org/10.1016/B978-0-12-746275-2.00019-7

Woodruff, C., Peck, G. y Banker, G. (1972). Effect of environmental conditions and polymer ratio on water vapor transmission through free plasticized cellulose films. Journal. Pharmaceutical Sciences, 61, 19561959. DOI: https://doi.org/10.1002/jps.2600611214C 